\title{
Estudio experimental de la osteosustitución con biomateriales cerámicos formulados como cementos óseos ${ }^{\#}$
}

\author{
Experimental study of the bone substitution with ceramic biomaterials formulated \\ as bone cements
}

\author{
E S Sanzana ${ }^{1 *}$, M P Ginebra ${ }^{2}$, P A Torres ${ }^{1}$, J A Planell ${ }^{2}$, M E Navarro ${ }^{2}$ \\ ${ }^{1}$ Laboratorio de Cirugía Experimental, Departamento de Cirugía, Facultad de Medicina, Universidad de Concepción, \\ Campus Universitario s/n, 4070106 Concepción, Chile. \\ ${ }^{2}$ Centre de Recerca en Enginyeria Biomèdica, Departament de Ciència dels Materials i Enginyeria Metal.lúrgica, \\ Universitat Politècnica de Catalunya, Diagonal 647, 08028 Barcelona, España.
}

\begin{abstract}
SUMMARY
Ceramic biomaterials have interesting biological properties that can be used in bone substitution. In this study two calcium phosphate bone cements were compared to cancellous bone autograft. A bone cavitary defect of $6 \mathrm{~mm}$ in diameter was carried out in the right distal femoral methaphyses of 36 male rabbits Oryctolagus cuniculi. The animals were divided into 3 groups of 12 rabbits receiving $\alpha$ tricalcium phosphate cement (TPC), monocalcium phosphate cement (MPC) and autologous bone (CON) as implants. The radiological and histological studies showed a correct substitution of both biomaterials with new bone. The histomorphometric study revealed that the bone neoformation obtained with the two cements at 4 and 12 weeks is equivalent to the bone generated by the bone graft. There were no significant differences in the reabsorption of the materials. Finally, this work concluded that the calcium phosphate bone cements are osteoconductive, osteotransductive and biocompatible materials which behave as bone substitutes.
\end{abstract}

Palabras clave: fosfatos de calcio, cementos óseos.

Key words: calcium phosphate, bone cements.

\section{INTRODUCCION}

La pérdida de tejido óseo es un evento frecuente en la traumatología y ortopedia, tanto humana como veterinaria, de tal manera que el implante preferido para el tratamiento de las soluciones de continuidad del hueso corresponde al propio tejido óseo, ya sea proveniente del mismo, o de otro individuo. El autoinjerto esponjoso es probadamente el mejor sustitutivo óseo en la clínica humana, sin embargo, tiene como limitantes la reducida cantidad de tejido disponible y la potencial morbilidad de los sitios dadores (Arrington y col 1996, Goulet y col 1997). Alternativamente, es posible disponer del aloinjerto óseo, ya sea congelado, criopreservado o liofilizado; pero, para su obtención y utilización, se requiere contar con un banco de tejidos y un número apropiado de donantes. Por lo demás, su empleo siempre conlleva el riesgo potencial de transmisión de enfermedades al receptor (Berrey y col 1990, Segur y col 1998).

Aceptado: 31.10.2006.

\# Financiado por los proyectos CICYT MAT 2002-04297 (España) y DIUC 205.081.026-1.0 (Chile)

* Casilla 60 C, Concepción, Chile, Fax: +56-41-2954416, Correo-e: esanzana@udec.cl
En los grandes defectos óseos diafisarios se puede practicar la técnica del transporte óseo, que a través de la osteogénesis por distracción es capaz de resolver exitosamente amplias pérdidas tisulares óseas (Ilizarov 1989). También es posible utilizar injertos óseos vascularizados que consiguen el mismo fin aprovechando las técnicas microquirúrgicas (Taylor y col 1975). No obstante, ninguno de estos dos métodos es aplicable para tratar defectos cavitarios.

Debido a las conocidas limitaciones que ostentan los injertos óseos, numerosos grupos de investigadores han estudiado variados biomateriales que pueden tener utilidad como sustitutivos óseos, sobresaliendo especialmente las cerámicas de fosfato de calcio, dado que detentan reconocidas propiedades osteoconductivas y son capaces de otorgar un andamiaje que en forma progresiva es sustituido por tejido óseo diferenciado (Hench 1998, LeGeros 2002). A este grupo pertenecen los cementos óseos basados en fosfatos de calcio.

La formulación de los biomateriales cerámicos como cementos óseos permite conseguir implantes moldeables, adaptables e inyectables, que se encuentran constituidos por una fase en polvo, que al ser mezclada con una solución acuosa dan lugar a la formación de una pasta plástica que posteriormente constituye un cuerpo sólido poroso (Le Geros y col 1982, Brown y Chow 1983). 
Los cementos óseos de fosfatos de calcio son capaces de fraguar en contacto con los fluidos orgánicos sin experimentar fenómenos de descohesión ni contracción, sus tiempos de fraguado y endurecimiento son reducidos, generan reacciones isotérmicas o exotérmicas limitadas, carecen de toxicidad local o general, detentan propiedades bioactivas, proporcionan resistencia mecánica en el sitio de implantación y el producto de su reacción es, habitualmente, una hidroxiapatita de rango nanométrico muy similar a la biológica. Estas características permiten postular estos materiales como sustitutivos óseos, como refuerzos biológicos para la osteosíntesis de fracturas y como cementos para la fijación de endoprótesis articulares (Ginebra y col 1994, 1997, 1998).

En 1993, Driessens y col, del Departament de Ciència dels Materials i Enginyeria Metal.lúrgica de la Universitat Politècnica de Catalunya, elaboraron un cemento de fosfato $\alpha$ tricálcico (TPC), que posteriormente fue optimizado por Ginebra y col (1994) para su aplicación en cirugía ósea y cuyo producto final es hidroxiapatita deficiente en calcio. En 1996 Driessens y col caracterizaron un cemento de fosfato monocálcico (MPC), formulado para generar como producto final de su reacción, hidroxiapatita precipitada, la que muestra una mayor solubilidad.

Al proyectar este trabajo experimental, nos propusimos como objetivo principal estudiar la regeneración ósea promovida in vivo por dos cementos óseos de fosfatos de calcio, en defectos cavitarios metafisarios femorales, comparándolos con el autoinjerto óseo esponjoso fresco.

\section{MATERIAL Y METODOS}

Se emplean como animales de experimentación 36 conejos machos albinos de la raza Nueva Zelanda, Oryctolagus cuniculi, con un peso comprendido entre 3.000 y 4.000 gramos y una edad entre 4 y 6 meses, los que se intervienen quirúrgicamente creando un defecto óseo cavitario de $6 \mathrm{~mm}$ de diámetro centrado en la metáfisis distal del fémur derecho (Katthagen 1986).

Se estudian 2 biomateriales cerámicos que corresponden a cemento de fosfato $\alpha$ tricálcico (TPC) y cemento de fosfato monocálcico (MPC), los que se comparan con un grupo control positivo implantado con autoinjerto óseo esponjoso fresco $(\mathrm{CON})$ que se obtiene del fémur contralateral.

Los animales de experimentación se distribuyen en 3 grupos de 12 conejos cada uno, los que a su vez se dividen en 2 subgrupos de 6 ejemplares, conformando un total de 6 subgrupos, en función del implante recibido y el tiempo de estabulación previo a la eutanasia.

Los conejos se anestesian con una combinación de 3 fármacos administrados por vía subcutánea; Hidrocloruro de Ketamina (Ketostop ${ }^{\circledR}$ ), en dosis de $35 \mathrm{mg} / \mathrm{kg}$ de peso, Clorhidrato de Xilazina 2\% (Xilazina ®), en dosis de 18 $\mathrm{mg} / \mathrm{kg}$ de peso y Acepromazina Maleato 1\% (Pacifor ®), en dosis de $1 \mathrm{mg} / \mathrm{kg}$ de peso. La profilaxis antibiótica se realiza con Enrofloxacino (Baytril 5\% ®) en dosis de 5 $\mathrm{mg} / \mathrm{kg}$ de peso, administrado por vía intramuscular al inicio del procedimiento anestésico.

Los cementos se preparan mediante la mezcla de sus componentes sólido en polvo y líquido hasta obtener una pasta plástica que se deja reposar entre 1 y 5 minutos. Entre los 5 y 6 minutos se inyecta el cemento con jeringa en el defecto óseo. Entre los 6 y 12 minutos se mantiene la herida operatoria abierta para permitir la aireación del material implantado y a los 12 minutos se procede a suturar el abordaje.

La analgesia posoperatoria se efectúa con Ketoprofeno (Ketofen $1 \%$ (R) en dosis de $2 \mathrm{mg} / \mathrm{kg}$ de peso administrado por vía intramuscular, cuya primera dosis se coloca al inicio de la intervención quirúrgica y luego se administra cada 12 horas hasta completar 7 días.

La eutanasia de los animales de experimentación se realiza con el ejemplar previamente sedado con Clorhidrato de Xilazina 2\% (Xilazina ${ }^{\circledR}$ ), administrando una sobredosis endovenosa de Tiopental Sódico 2\% (Pentothal $®$ ).

La obtención de los especímenes óseos se realiza mediante el abordaje lateral longitudinal del muslo derecho del conejo, lo que permite extraer el fémur completo desarticulándolo a nivel de cadera y rodilla.

Las muestras óseas son sometidas en primer lugar a una valoración radiológica, para continuar su preparación con técnica para hueso no descalcificado y obtener cortes histológicos de $5 \mu \mathrm{m}$ que se tratan con la tinción de tricrómico de Goldner. Seguidamente, se efectúa un estudio histológico y un análisis histomorfométrico.

Las mediciones histomorfométricas se realizan empleando un microscopio (Zeiss ${ }^{\circledR}$ Axioplan 2) provisto de una cámara digital (Nikon ® DXM 1200) y el análisis de las imágenes se efectúa con el programa informático Image-Pro ${ }^{\circledR}$ Plus 4.5. De esta manera, se determinan el Area de Implantación (AI), el Area Trabecular Total (ATT) y el Area de Biomaterial No Reabsorbido (ABNR), medidas que se expresan en $\mathrm{mm}^{2}$.

El Area de Implantación (AI) debería medir teóricamente $28,274 \mathrm{~mm}^{2}$ correspondientes al cálculo del área de un círculo con un radio de $3 \mathrm{~mm}$, no obstante, la medición real suele no ser coincidente con este valor, debido a que los cortes histológicos no son siempre, perfectamente perpendiculares.

El Area Trabecular Total (ATT) representa la superficie que ocupan las trabéculas óseas neoformadas en el defecto cavitario creado e indica la cantidad de tejido óseo presente en su interior.

El Area de Biomaterial No Reabsorbido (ABNR) corresponde a la superficie ocupada por los restos de los cementos óseos implantados en el defecto cavitario experimental.

La normalidad de las variables cuantitativas se verifican con las pruebas de Kolmogorov-Smirnov y ShapiroWilk, aceptándose el supuesto de normalidad si $\mathrm{P}>0,05$. 
Para la comparación de dichos resultados se utiliza el análisis de la varianza (ANOVA), realizándose un establecimiento de contrastes mediante el método de Tukey HSD en los casos con resultados estadísticamente significativos. Se acepta que existe significación estadística cuando $\mathrm{P}<0,05$. Todos los análisis estadísticos se realizan con el programa informático SPSS 11.0 para Windows.

\section{RESULTADOS}

Los resultados de este trabajo experimental se han obtenido valorando las muestras óseas correspondientes a 33 animales de experimentación útiles, dado que se descartó del estudio un ejemplar de cada uno de los 3 subgrupos estabulados por 4 semanas, debido a complicaciones intercurrentes.

La evaluación radiológica ha permitido constatar que los 3 implantes se osteointegraron, ya sea en forma completa, como en el caso del autoinjerto óseo, o de manera parcial, como ocurrió con los 2 cementos de fosfato de calcio (figura 1).

El estudio histológico ha mostrado que ambos cementos óseos desarrollaron un enlace directo con el tejido óseo del defecto cavitario de la metáfisis femoral. De esta manera, se ha observado el desarrollo de una neoformación ósea homogénea, progresiva y centrípeta en todas las muestras estudiadas, encontrando que a las 12 semanas persisten restos de los materiales implantados en el área central de los defectos, de tal forma que ninguno de los 2 cementos de fosfato de calcio se ha reabsorbido en forma completa.

El análisis microscópico de los cementos en el primer período de estudio ha mostrado la presencia de un

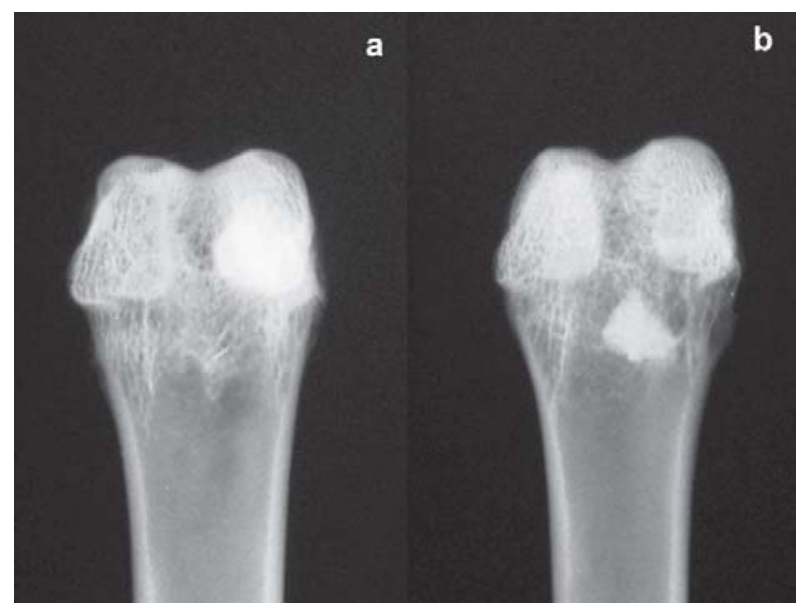

Figura 1. Evaluación radiológica del TPC a las 4 semanas de implantación (a) y a las 12 semanas con neoformación ósea y reabsorción parcial (b).

Radiologic evaluation of TPC to the 4 weeks of implantation (a) and to the 12 weeks with bone neoformation and partial resorption (b). tejido óseo neoformado de aspecto inmaduro, con abundante celularidad y la constitución de un tejido osteoide de grosor moderado e irregular en continuidad con el hueso pericavitario (figura 2).

En el estudio histológico del segundo período se ha observado principalmente la conformación de numerosas trabéculas óseas, bien conectadas, envolviendo y rodeando a los restos de los implantes en proceso de biodegradación (figura 3).

El análisis microscópico del autoinjerto óseo en el primer período de estudio no ha permitido diferenciar el hueso esponjoso implantado respecto del tejido óseo neoformado. En el segundo período se han verificado principalmente fenómenos de remodelación y calcificación de las numerosas trabéculas óseas que se han constituido en el defecto injertado.

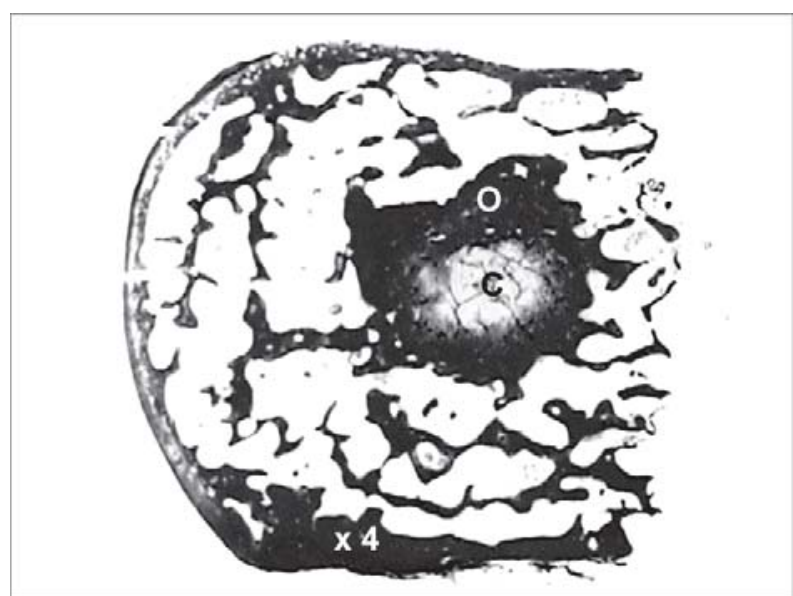

Figura 2. Estudio microscópico panorámico del MPC a las 4 semanas, con la presencia de osteoide $(\mathrm{O})$ y cemento óseo (C).

Panoramic microscopic study of MPC to the 4 weeks, with the osteoid presence $(\mathrm{O})$ and bone cement $(\mathrm{C})$.

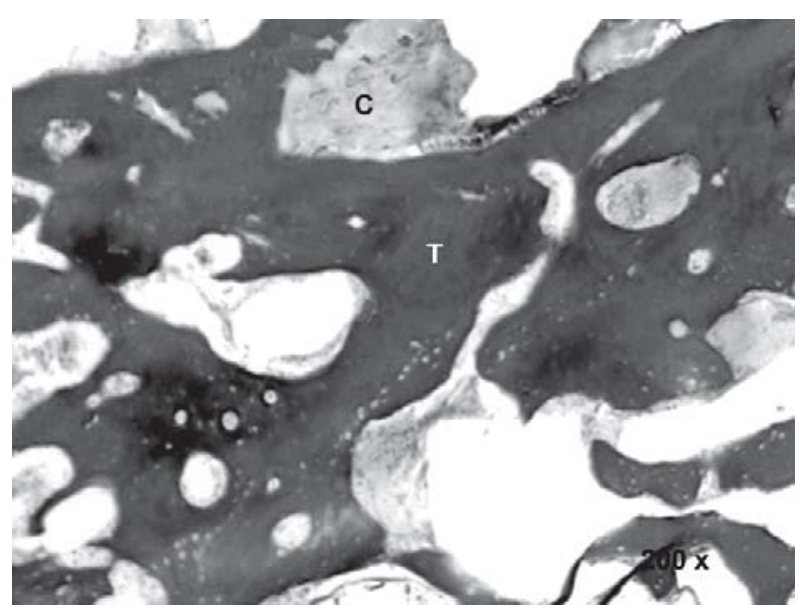

Figura 3. Análisis histológico del MPC a las 12 semanas, con la presencia de trabéculas en remodelación $(\mathrm{T})$ y restos de cemento $(\mathrm{C})$.

Histologic analysis of MPC to the 12 weeks, with remodeling trabeculae presence $(\mathrm{T})$ and cement remains $(\mathrm{C})$. 
El estudio histomorfométrico comparativo de medias que se ha realizado, tanto a las 4 como a las 12 semanas, entre los distintos grupos de estudio y el Area de Implantación (AI), Area Trabecular Total (ATT) y Area de Biomaterial No Reabsorbido (ABNR), no ha mostrado diferencias estadísticamente significativas $(\mathrm{P}<0,05)$ (figura 4).

\section{DISCUSION}

La sustitución del hueso continúa siendo un problema no resuelto, situación que ha favorecido enormemente el desarrollo de variadas líneas de investigación que han estudiado la formulación, caracterización y elaboración de numerosos materiales que pueden tener utilidad en el ámbito de la regeneración ósea, destacando muy particularmente los biomateriales cerámicos por su similitud estructural con el componente mineral del hueso (Hench 1998, LeGeros 2002).

Los cementos óseos de fosfatos de calcio se comportan como osteosustitutivos dado que son capaces de conducir la neoformación ósea y que se integran al hueso mediante el mecanismo de osteotransducción. De este modo el material sólido poroso resultante de la mezcla de la fase en polvo y la fase líquida del cemento entra a formar parte del proceso de neoformación ósea, por lo que su degradación es activa mediante la acción de los osteoclastos. Así, el concepto de osteotransducción se puede definir como la fijación biológica y bioactiva de un material con biodegradabilidad selectiva activa y biotransformación. Destaca la ausencia de interfaz en la unión entre el cemento y el hueso que el estudio histológico de este trabajo ha verificado, tanto para el cemento de fosfato $\alpha$ tricálcico (TPC), como para el cemento de fosfato monocálcico (MPC) (Driessens 1993, 1998).

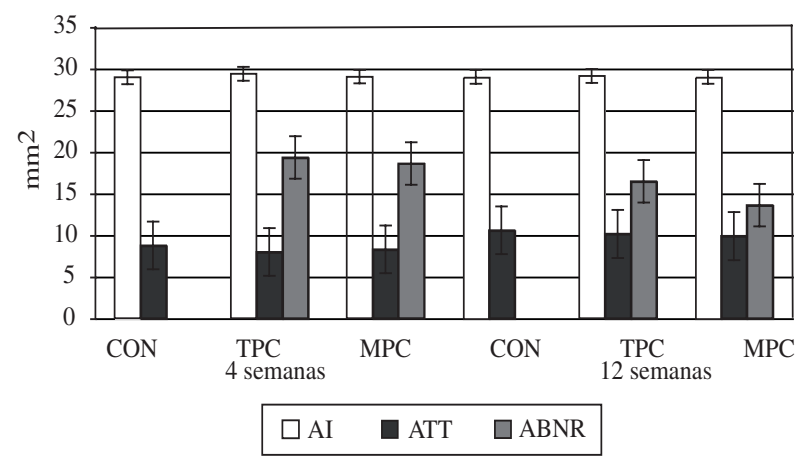

Figura 4. Mediciones histomorfométricas obtenidas a las 4 y 12 semanas, del Area de Implantación (AI), Area Trabecular Total (ATT) y Area de Biomaterial No Reabsorbido (ABNR), en los grupos control (CON), cemento de fosfato $\alpha$ tricálcico (TPC) y cemento de fosfato monocálcico (MPC).

Histomorphometric measurements obtained to the 4 and 12 weeks of Area of Implantation (AI), Total Trabecular Area (ATT) and Area of Not Reabsorbed Biomaterial (ABNR), in the control (CON), $\alpha$ Tricalcium Phosphate Cement (TPC) and Monocalcium Phosphate Cement (MPC) groups.
Un cemento óseo ideal debería ser capaz de reabsorberse a la misma velocidad que se desarrolla el tejido óseo neoformado encargado de su reemplazo, no obstante, la mayoría de estos materiales posee una cinética de reabsorción lenta, lo que representa una desventaja debido a que la resistencia mecánica que aportan, en el mejor de los casos, es igual a la del hueso esponjoso o equivalente a un $20 \%$ de la del hueso cortical. El TPC presenta una resistencia mecánica máxima de 40 megapascales (MPa) a las 40 horas de su fraguado, en cambio el MPC aporta unos valores máximos de resistencia a la compresión que promedia los 12 $\mathrm{MPa}$ a las 64 horas. Por lo anterior, estos materiales no son aptos para ser implantados en áreas óseas sometidas a solicitaciones mecánicas importantes y sus indicaciones actuales se mantienen limitadas al relleno de defectos óseos cavitarios y como refuerzos de dispositivos de osteosíntesis (Ginebra y col 1994, Driessens y col 1996).

El fraguado de los cementos de fosfato de calcio genera, habitualmente, una reacción isotérmica, aunque algunas formulaciones pueden producir efectos exotérmicos restringidos, con alzas de temperatura de alrededor de unos $10{ }^{\circ} \mathrm{C}$, hecho que previene la lesión potencial de los tejidos vecinos. Lo anterior ha despertado el interés por destinar estos biomateriales cerámicos a la cementación de endoprótesis articulares, como una manera de evitar la gran reacción exotérmica, de hasta $133{ }^{\circ} \mathrm{C}$, que genera el cemento de polimetilmetacrílato empleado usualmente (Fernández y col 1995, Ginebra y col 1995).

De acuerdo con sus distintas formulaciones, se puede clasificar al TPC como un material de reabsorción intermedia y al MPC como de reabsorción rápida, siendo esperable diferencias en los resultados histológico e histomorfométrico (Driessens 1993, 1996). Sin embargo, el estudio histológico ha revelado que ambos cementos promueven la formación de un tejido osteoide de similares características, cuya maduración es lenta, de tal forma que a las 12 semanas se encuentra un tejido óseo trabecular menos remodelado que el del grupo control. Los resultados histomorfométricos muestran que la tasa de neoformación ósea generada por ambos cementos es equivalente a la del autoinjerto óseo esponjoso fresco, tanto a las 4 como a las 12 semanas y la tasa de reabsorción no presenta diferencias estadísticamente significativas para ambos biomateriales. Estos resultados son concordantes con los obtenidos en estudios previos que han evaluado el comportamiento in vivo de los cementos óseos de fosfatos de calcio, en la osteosustitución en animales de experimentación (Driessens 1995, Jansen 1996 , Torner 2001, Ooms 2002, Ooms 2003).

La biocompatibilidad de los cementos de fosfatos de calcio es un factor de mucha importancia para disponer su empleo como sustitutivos óseos efectivos. Los estudios de toxicidad local o general, de mutagenicidad 
y carcinogénesis han resultado negativos y los productos de degradación de estos materiales aportan calcio y fósforo para la neoformación ósea desarrollada por los osteoblastos. Sin embargo, en algunos estudios se ha detectado la presencia de algunas reacciones inflamatorias leves alrededor de los implantes que parecen tener relación con la formulación y composición particular de ciertos cementos. En este estudio no hemos detectado manifestaciones inflamatorias ni de toxicidad frente a los 2 cementos óseos de fosfatos de calcio estudiados (Yoshimine y col 1993, Jansen y col 1996, Yuan y col 2000).

Además de su capacidad para sustituir el tejido óseo en las soluciones de continuidad del hueso, resulta de mucho interés la utilidad de los cementos óseos de fosfatos de calcio como materiales liberadores de sustancias (Drug Delivery System), dado que su degradación progresiva permite el pasaje lento de variados fármacos al interior del hueso (Hamanishi y col 1996, Sasaki e Ishii 1999). En forma paralela, diferentes estudios biomecánicos efectuados en esqueletos de cadáver han demostrado que estos biomateriales pueden favorecer y facilitar la fijación de determinadas fracturas (Stankewich y col 1996, Higgins y col 2002). Por otro lado, ya se cuenta con experiencias clínicas que describen el empleo de algunos cementos fosfocálcicos para mejorar la consolidación de ciertas fracturas metafisarias, cuyos resultados preliminares en series limitadas de casos aparecen como promisorios (Larson y Bauer 2002).

En conclusión, el presente trabajo experimental ha hecho posible corroborar las propiedades biológicas de los cementos óseos de fosfatos de calcio in vivo, confirmando su condición de materiales osteoconductivos, osteotransductivos, reabsorbibles, degradables, biocompatibles y osteointegrables. Lo anterior permite afirmar que estos biomateriales cerámicos son útiles para la sustitución ósea, por lo que es esperable una ampliación y masificación de su empleo clínico.

\section{RESUMEN}

Los biomateriales cerámicos presentan interesantes propiedades biológicas, por lo que pueden ser utilizados en la sustitución ósea. En este estudio fueron comparados dos cementos óseos de fosfato de calcio con el autoinjerto óseo esponjoso. Se realizó un defecto cavitario de $6 \mathrm{~mm}$ en la metáfisis femoral distal derecha de 36 conejos machos Oryctolagus cuniculi. Los animales fueron divididos en 3 grupos de 12 conejos, que recibieron como implantes cemento de fosfato $\alpha$ tricálcico (TPC), cemento de fosfato monocálcico (MPC) y autoinjerto óseo (CON). Los estudios radiológico e histológico han mostrado una correcta sustitución de ambos biomateriales por hueso neoformado. El estudio histomorfométrico ha revelado que la neoformación ósea obtenida con los 2 cementos, tanto a las 4 como las 12 semanas, es equivalente a la generada por el injerto óseo. No se han encontrado diferencias significativas en la reabsorción de los materiales. Finalmente, este trabajo ha concluido que los cementos óseos de fosfato de calcio son materiales osteoconductivos, osteotransductivos y biocompatibles que se comportan como sustitutivos óseos.

\section{REFERENCIAS}

Arrington ED, WJ Smith, HG Chambers, AL Bucknell, NA Davino. 1996. Complications of iliac crest bone graft harvesting. Clin Orthop 329, 300-309.

Berrey BH, CF Lord, MC Gebhard, HJ Mankin. 1990. Fractures in allografts. Frequency, treatment and end-results. J Bone Joint Surg 72 A, 825-839.

Brown WE, LC Chow. 1983. A new calcium phosphate setting cement. J Dent Res 62, 672.

Driessens FCM, MG Boltong, JA Planell, O Bermúdez, MP Ginebra, E Fernández. 1993. A new apatitic calcium phosphate bone cement: preliminary results. Bioceramics 6, 469-473.

Driessens FCM, MG Boltong, MI Zapatero, RMH Verbeek, W Bonfield, O Bermúdez, E Fernández, MP Ginebra, JA Planell. 1995. In vivo behavior of three calcium phosphate cements and a magnesium phosphate cement. J Mater Sci Mater Med 6, 272-278.

Driessens FCM, EAP De Maeyer, E Fernández, MG Boltong, G Berger, RMH Verbeek, MP Ginebra, JA Planell. 1996. Amorphous calcium phosphate cements and their transformation into calcium deficient hydroxyapatite. Bioceramics 9, 231-234.

Driessens FCM, JA Planell, MG Boltong, I Khairoun, MP Ginebra. 1998. Osteotransductive bone cements. Proc Instn Mech Engrs [H] 1998; 212, 427-435.

Fernández E, MP Ginebra, O Bermúdez, MG Boltong, FCM Driessens, JA Planell. 1995. Dimensional and thermal behavior of calcium phosphate cements during setting compared to PMMA bone cements. J Mat Sci Let 14, 4-5.

Ginebra MP, E Fernández, MG Boltong, O Bermúdez, JA Planell, FCM Driessens. 1994. Compliance of an apatitic calcium phosphate cement with short-term clinical requirements in bone surgery, orthopaedics and dentistry. Clin Mat 17, 99-104.

Ginebra MP, E Fernández, FCM Driessens, M Boltong, J Muntasell, J Font, JA Planell. 1995. The effects of temperature on the behavior of an apatitic calcium phosphate cement. J Mater Sci Mater Med 6, 857-860.

Ginebra MP, E Fernández, EAP De Maeyer, RMH Verbeek, MG Boltong, J Ginebra, FCM Driessens, JA Planell. 1997. Setting reaction and hardening of an apatitic calcium phosphate cement. J Dent Res 76, 905-912.

Ginebra MP, E Fernández, FCM Driessens, JA Planell. 1998. The effect of $\mathrm{Na}_{2} \mathrm{HPO}_{4}$ addition on the setting reaction kinetics of an $\alpha$-TCP cement. Bioceramics 11, 243-246.

Goulet JA, LE Senunas, GL DeSilva, ML Greenfield. 1997. Autogenous iliac crest bone graft. Complications and functional assesment. Clin Orthop 339, 76-81.

Hamanishi C, K Kitamoto, S Tanaka, M Otsuka, Y Doi, T Kitahashi. 1996. A self-setting TTCP-DCPD apatite cement for release of vancomycin. J Biomed Mater Res 33, 139-143.

Hench LL. 1998. Bioceramics. J Am Ceram Soc 81, 1705-1728.

Higgins TF, SD Dodds, SW Wolfe. 2002. A biomechanical analysis of fixation of intra-articular distal radial fractures with calciumphosphate bone cement. J Bone Joint Surg 84 A, 1579-1586.

Ilizarov GA. 1989. The tension-stress effect on the genesis and growth of tissues. Part I. The influence of stability of fixation and softtissue preservation. Clin Orthop 238, 249-281.

Jansen JA, JE De Ruijter, HG Schaeken, JPCM Van der Waerden, JA Planell, FCM Driessens. 1995. Evaluation of tricalciumphosphate / hydroxiapatite cement for tooth replacement: an experimental animal study. J Mater Sci Mater Med 6, 653-657.

Katthagen BD. 1986. Bone regeneration with bone substitutes. An animal study. Springer Verlag. Berlin, Alemania.

Larsson S, TW Bauer. 2002. Use of injectable calcium phosphate cement for fracture fixation: a review. Clin Orthop 395, 23-32. 
LeGeros RZ, A Chohayeb, A Shulman. 1982. Apatitic calcium phosphate: possible restorative materials. J Dent Res 61, 343.

LeGeros RZ. 2002. Properties of osteoconductive biomaterials: calcium phosphates. Clin Orthop 395, 81-98.

Ooms EM, JG Wolke, JP Van der Waerden, JA Jansen. 2002. Trabecular bone response to injectable calcium phosphate (Ca-P) cement. J Biomed Mater Res 61, 9-18.

Ooms EM, JG Wolke, MT Van de Heuvel, B Jeschke, JA Jansen. 2003. Histological evaluation of the bone response to calcium phosphate cement implanted in cortical bone. Biomaterials 24 989-1000.

Sasaki S, Y Ishii. 1999. Apatite cement containing antibiotics: efficacy in treating experimental osteomyelitis. J Orthop Sci 4, 361-369.

Segur JM, S Suso, S García, A Combalía, R Ramón. 1998. Bone allograft contamination in multiorgan and tissue donors. Arch Orthop Trauma Surg 118, 156-158.
Stankewich CJ, MF Swiontkowski, AF Tencer, DN Yetkinler, RD Poser. 1996. Augmentation of femoral neck fracture fixation with an injectable calcium-phosphate bone mineral cement. J Orthop Res 14, 786-793.

Taylor GI, GD Miller, FJ Ham. 1975. The free vascularized bone graft: A clinical extension of microvascular techniques. Plast Reconstr Surg 55, 533-544.

Torner P. 2001. Reparació del teixit ossi mitjançant osteotransducció amb ciment de fosfat càlcic. Estudi experimental. PhD Thesis Dept. of Surgery. Faculty of Medicine. University of Barcelona.

Yoshimine Y, A Akamine, M Mukai. 1993. Biocompatibility of tetracalcium phosphate cement when used as a bone substitute. Biomaterials 14, 403-406

Yuan H, Y Li, JD De Bruijn, K De Groot, X Zhang. 2000. Tissue responses of calcium phosphate cement: a study in dogs. Biomaterials 21, 1283-1290. 